\title{
IN MEMORIAM LIONEL BIER
}

Prof. Lionel Bier ist am 4. März 2004 im Roosevelt Hospital in New York gestorben. In einem kurzen und harten Kampf gegen die Krankheit, den er im Vertrauen auf seine durch lebenslange sportliche Aktivität gute Kondition voller Optimismus führte, wurde er von der Leukämie letztlich besiegt.

Lonny Bier hatte zum Jahresende 2003 seine Lehrtätigkeit am Brooklyn College aufgegeben, um sich künftig ausschließlich seiner wissenschaftlichen Tätigkeit als Bauforscher in Aphrodisias und Ephesos zu widmen - an der Seite Maria Aurenhammers in Wien. Es war ihm nicht vergönnt, diesen neuen Lebensabschnitt anzutreten und seine weit gediehenen Untersuchungen an den Bouleuterien von Aphrodisias und Ephesos zu vollenden und zu publizieren.

Lionel Bier wurde am 28. Mai 1942 in Brooklyn geboren, dort absolvierte er Schule und College und dort lehrte er später als Professor. Seine akademischen Grade erwarb er am Institute of Fine Arts der New York University (M.A. 1968, Ph.D. 1979), dem er sein

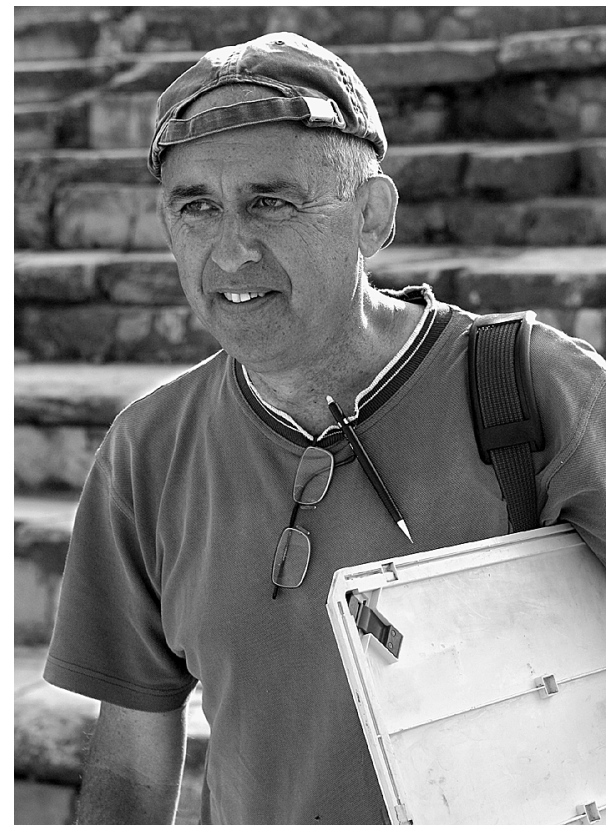

Lionel Bier $(\dagger)$ Leben lang eng verbunden blieb. Die Lehrtätigkeit am Brooklyn College begann er bereits 1975 als Dissertant, seit 1988 lehrte er als Professor die gesamte Kunstgeschichte und seine Spezialgebiete, die Kunst und Architektur des Nahen Ostens, Ägyptens und des hellenischen Raumes. 1979 las er an der Columbia University, Department of Art History and Archeology, »The Architecture of Iran in the Sasanian and Early Islamic Periods « und 1984 am Institute of Fine Arts der N. Y. University »The Graphic Documentation of Architecture: Surveying and Drafting for Archeologists and Architectural Historians«. Seit Beginn seines Studiums nahm er regelmäßig an zahlreichen archäologischen Grabungen im Nahen Osten und in Westkleinasien als Topograph, Architekt und Bauforscher teil (Knidos 1967-1969; Koruçutepe in der Türkei 1969; Taposiris Magna in Ägypten 1975; Dokumentation sassanidischer und frühislamischer Monumente im Iran 1975-1976, Survey im unteren Morava-Tal in Jugoslawien 1981; Balboura in der Türkei 1986, 1987 und 1990; Bauaufnahme der Abtei Nôtre Dame, Jumièges in Frankreich 1997; Aphrodisias 1992-2003; Ephesos 1997-2003).

Die Forschungen im Iran konnte er 1975-1976 mit Hilfe des Fulbright-Hays-Stipendiums durchführen, daraus resultierte auch seine Dissertation $»$ Sarvistan. A study in Early Iranian Architecture «, Monographs on Fine Arts 45 (1986). Für eine erste Version dieser Arbeit erhielt er 1979 den »James C. Healey Award for Outstanding Dissertation in the Humanities« der N. Y. University. Seine Tätigkeiten im Iran lieferten außerdem die Grundlage für zahlreiche weitere Artikel (A Second Hittite Relief at Ivriz, JNES 35, 1976, 115-126; Sasanian Palaces in Perspective, Archaeology 35, 1982, 29-36; A Sculpted Building Block from Istakhr, AMI 16, 1983, 305-314; The Masjid-i Sang near Dārāb and the Mosque of Shahr-i İj: Rock-cut Architecture of the Il-Khanid Period, Iran 24, 1986, 117-130; Notes on Mihr Narseh's bridge near Firuzabad, AMI 16, 1986, 263-268; The Sasanian Palaces and their Influence in Early Islam, Ars Orientalis 23, 1993, 57-66; Sarvistan Reconsidered, in: Leaving no Stones Unturned: Essays on the Ancient Near East and Egypt in Honor of Donald P. Hansen [2002] 43-51) sowie mehrere Rezensionen (D. Whitcomb, Before Roses and Nightingales. Excavations at Qasr-I Abu Nasr, Old 
Shiraz [1985], Journal of the American Oriental Society 107, 1987, 814-815; C. Adle [Hrsg.], Art et Société dans le monde Iranien, Institut Francais d'Iranologie de Téhéran, Bibliothèque Iranienne 26 [1982], Journal of the American Oriental Society 109, 1989, 302-303; W. Kleiss, Die Entwicklung von Palästen und palastartigen Wohnbauten in Iran, ZA 79, 1989, 300-301; R. Boucharlat - O. Lecomte, Fouilles de Tureng Tepe sous la direction de Jean Deshayes, I. Les Périodes Sassanides et Islamiques, Mémoires 74 [1987], Journal of the American Oriental Society 110, 1990, 347-349; M. Azarnoush, The Sasanian Manor House at Hajiabad, Iran [1994], BiOr 53, 1996, 836-838) und Lexikonartikel (»Fars«, »Naqsh-I Rustan«, »Paikuli«, »Takht-I Sulaiman«, »Taq-I Bustan«, in: Dictionary of the Middle Ages; »Iwan«, »Sarvistan«, in: The Dictionary of Art).

Später galt sein Interesse zunehmend der archäologischen Bauforschung an antiken Stätten der Türkei. Zunächst untersuchte er die Theaterbauten von Balboura (The Lower Theatre at Balboura, AnatSt 40, 1990, 69-79; The Upper Theatre at Balboura, AnatSt 44, 1994, 27-46), dann arbeitete er als Topograph in Aphrodisias, wo er auch die detaillierte Bauuntersuchung des Odeions/Bouleuterions durchführte (The Bouleuterion at Aphrodisias, Aphrodisias Papers IV [in Druck]). Im Rahmen einer vergleichenden Studie an überdachten Versammlungsbauten erwachte sein Interesse an dem Bouleuterion am Staatsmarkt in Ephesos, dessen Bearbeitung er 1997 mit einer systematischen Bauaufnahme und Bauanalyse begann (The Bouleuterion at Ephesos: Observations for a New Survey, in: P. Scherrer - H. Taeuber - H. Thür [Hrsg.], Steine und Wege. Festschrift D. Knibbe, SoSchrÖAI 32 [1999] 7-18). Neben seiner Lehrtätigkeit und seinen Forschungen hielt Lonny Bier zahlreiche Vorträge über sassanidische Kunst und Architektur sowie in den letzten Jahren vorwiegend über seine architekturhistorischen Untersuchungen an den überdachten Theaterbauten von Aphrodisias und Ephesos.

Lonny Bier hatte den Umgang mit dem Zeichenstift schon als Kind bei seinem Vater, einem Schiffsbauingenieur, gelernt, das Talent zum Zeichnen und Entwerfen von Architektur war ihm offensichtlich angeboren. In Aphrodisias übernahm er die Aufgabe des Topographen, um den begonnenen Stadtplan zu erstellen, obwohl er den Umgang mit der hochtechnisierten geodätischen Totalstation autodidaktisch erlernen mußte, wobei ihm glücklicherweise der technische Spürsinn eines Schülers zu Hilfe kam. Auch das Handwerk der traditionellen Bauaufnahme, die Handzeichnung, erlernte er 'by doing' in höchster Perfektion, wie seine aussagekräftigen exakten und zugleich kunstvollen Zeichnungen, aber auch seine Lehrveranstaltung zu diesem Thema zeigen. Er suchte stets den wissenschaftlichen Disput über Bautechnik, Bedeutung und Interpretation baulicher Details und diskutierte unermüdlich seine neuesten Beobachtungen und deren Deutungsmöglichkeiten. Wie oft saß er frühmorgens - hellwach und voller Tatendrang - im Speisesaal des ephesischen Grabungshauses und wartete ungeduldig auf das Erscheinen und die - infolge der frühen Stunde manchmal noch reduzierte - Redebereitschaft fachkundiger Gesprächspartner. Seine Begeisterung für die Bauforschung und sein Enthusiasmus in der Entschlüsselung der Bauphasen des ephesischen Bouleuterions waren so groß, daß er allen widrigen Umständen - der gnadenlosen anatolischen Sommerhitze und den Besuchermassen - dieses Arbeitsplatzes zum Trotz seine Aufnahmen, Beschreibungen und Auswertungen im Alleingang in erstaunlich kurzer Zeit fertigstellte. Eine vollständige Bauaufnahme liegt so nicht nur für das Odeion/Bouleuterion in Aphrodisias, sondern auch für das Bouleuterion in Ephesos vor, so daß die Untersuchungen beider Bauwerke in Kürze und in seinem Sinne fertiggestellt und publiziert werden können.

Innerhalb des Ephesos-Teams entwickelten sich durch Lonnys unerschöpfliche Wißbegierde und Neugier für viele Fragestellungen, seine amerikanisch offene Art und sein stets für Diskussionen bereites herzliches Wesen Freundschaften, die durch Aufenthalte in Wien zwecks Archivstudien am Österreichischen Archäologischen Institut vertieft wurden - gewürzt mit dem Studium der Wiener 'Beisln' und Heurigen, Wanderungen und sportlichen Radtouren in der Wiener Umgebung. Die überaus herzliche Gastfreundschaft im Domizil Aurenhammer-Bier in Wien und insbesondere in Selçuk wurde so für zahlreiche 'Ephesier' zu einem hoch geschätzten Treff- und Fixpunkt innerhalb des Grabungslebens. 
Das Ephesos-Team hat mit Lionel Bier einen allseits geschätzten und anerkannten Bauforscher verloren, die Absenz dieses kompetenten Kollegen hinterläßt eine große Lücke; schmerzlicher noch fehlt der kultivierte, auch außerhalb des Faches gebildete und belesene Mensch, der außer seiner Muttersprache viele Fremdsprachen beherrschte, fließend deutsche Gedichte rezitierte, Türkisch und Persisch sprach. Viele Ephesier und Wiener Kollegen trauern mit Maria Aurenhammer, die den Partner verlor, um einen lieben Freund.

Doz. Dr. Hilke Thür

Institut für Kulturgeschichte der Antike, Österreichische Akademie der Wissenschaften, Bäckerstraße 13, A-1010 Wien

E-Mail: hilke.thuer@oeaw.ac.at

Abbildungsnachweis: Photo F. Krinzinger. 
